\section{Biorepositories and Biomarker Development: Regulatory Challenges}

\section{Abstract}

Biomarker development has become an indispensable tool in personalized medicine drug development. Health care costs expenditures for biologics therapeutics paired with individualized, personalized medicine is driving the need for biomarker development, which is the companion diagnostic medical device (CDx), that allows treating the right patient at the right time with the right drug or drug combination at the right dose. Early biomarker development may be facilitated by leveraging biorepositories or biobanks from human-derived specimen, representing the target disease. Legally authorized biorepositories or biobanks thus may provide a versatile "tool" to determine performance characteristics and/or analytical/ clinical validity of the in vitro diagnostic medical device, in the specific context of drug use prior to moving into clinical phase. Authorization of biobanks for research and commercial use originates from compliance to Declaration of Helsinki and in principle is based on national and international legislation, statutory requirements and informed consent provided by donors, while informed consent may be waived in some circumstances. Europe is currently facing a 5 year "grace period" with respect to transition of regulations for $C D x$ biomarker development, even though the new Regulation on In vitro Diagnostic Medical Devices (IVDR) has already come into effect May 26, 2017. The changing regulatory conditions and the availability of various biorepositories paired with new European unequivocal provisions for CDx biomarker development and conformity assessment procedures for approval (CE-marking) may allow to develop biomarker performance characteristics and/ or analytical/clinical validity even in the absence of contemporaneously sourced clinical trials specimen. This article sheds some light on regulatory challenges with respect to upcoming changes with the IVDR and illustrates the pitfalls associated with the CDx biomarker in the use of biorepositories.

Keywords: Biobank; Biorepository; Ethics; Biomarker; Polyomavirus; European regulations

\section{Hess RD* and Laaff $H$ \\ TPL Path Labs, Sasbacher Strabe 10, D-79111 Freiburg, Germany}

\section{Corresponding author: Hess RD}

hess@tpl-path-labs.com

TPL Path Labs, Sasbacher Strabe 10, D-79111 Freiburg, Germany.

Tel: 0049076145688658

Citation: Hess RD, Laaff H (2017)

Biorepositories and Biomarker Development: Regulatory Challenges. Biomark J. Vol.3 No.3:15.

\section{Biomarker Development}

Personalized medicine is the most progressing field in medical research addressing unmet medical need shifting the classical therapeutic approach based on anti-cancer agents in tumor treatment to the individualized, personalized medicine $[1,2]$. Increasingly understanding the etiology of tumorigenesis paired with unravelling molecular pathways has led to specifically targeting not only oncologic but also autoimmune and inflammatory maladies as well as immuno oncology conditions. According to FDA and Biomarker Definition Working group a biomarker is "a characteristic that is objectively measured and evaluated as an indicator of normal biological processes, pathogenic processes, or pharmacologic responses to a therapeutic intervention" $[3,4]$. Companion diagnostics (CDx, which are in vitro medical diagnostic devices) the specific biomarker development targeting protein, or DNA, or RNA tested for in the specific context of drug use have revolutionized personalized medicine dramatically. Cost-intensive monoclonal antibody therapeutic regimen has significantly contributed to high healthcare burden [5], while application of biomarkers could improve health care and contributes to reducing healthcare 
costs. The American Society of Clinical Oncology estimates that routinely testing people with colon cancer for mutations in the K-RAS oncogene would save at least US $\$ 600$ million a year $[6,7]$. This may necessitate to consider- among others-predictive as well as pharmacodynamics biomarker for selection of patients or pharmacodynamic effects of a drug, respectively [8]. Unfortunately, the health care systems have not yet fully paved the way to reimbursement of novel biomarker-based medicinal laboratory tests for personalized medicine [9], not necessarily providing an additional driver for the biomarker development.

As an example, the immunohistochemistry-based Human epidermal growth factor receptor 2 (HER-2) - one of the first predictive biomarker in human breast cancer [10] - was shown to be a suitable method for using HER-2/neu status to predict response to antibody therapeutic Herceptin-based therapy [11]. HER-2 also proved to be a specific target in HER2-positive patients suffering from metastatic adenocarcinoma of the stomach or gastroesophageal junction who have not received prior anticancer treatment for their metastatic disease. These patients can be treated with Herceptin in combination with capecitabine or 5-fluorouracil and cisplatin. Determination of HER2 overexpression is done by immunohistochemistry testing (IHC), while HER2 gene amplification utilizes a molecular biomarker, such as Fluorescence in situ Hybridisation (FISH) also including Silver-enhanced In situ Hybridisation (SISH) and applied as confirmatory testing [12]. Thus, there is clearly a patient added value in the application of biomarkers in the specific context of the drugs used, which in this case may be enhancing therapeutic efficacy with a drug combination.

\section{The value of a biorepository in biomarker development}

The OECD provides "Best Practice Guidelines" for the procurement and supply of human-derived tissues and materials for basic research and also in general and applied sciences, that constitute vital tools for clinical, health-related biotechnologies and the development of new drugs, diagnostic devices and therapies. As a prerequisite, legally authorized supply of humanderived materials requires highest quality standards, consistent traceability from the providers to the end user in accordance with national and international regulations, as well as ethical commitments where these are required to apply [13]. The early phase of a biomarker CDx development may be considered early exploratory biomarker which might be developed in research laboratories which are neither accredited nor certified to accepted regulatory GXP standards. In contrast, pivotal nonclinical and clinical studies have to be performed in compliance with GLP, GCP or "GCLP" [14] respectively. Exploratory studies are performed in order to identify the most appropriate target population in addition to the usual aims: to define dose, schedule, tumor type and line of therapy [15]. The availability of archived human-derived tissues and materials sourced from legally authorized biobanks may provide valuable tool in early biomarker development, the stage in CDx development prior to moving into clinical phase to demonstrate performance characteristics and/or clinical validity and clinical utility.
Simon et al., 2009 discussed potential "analytical concerns", which address reproducibility and robustness and additional factors that minimize variation in the context of pre-analytical factors, that includes the collection of tissues, its processing and storage conditions and also method-specific conditions. Whenever archived specimens are used the archived sample shall reflect true clinical settings [16]. Pre-analytical processing steps are subject to variability especially utilizing archived Formalin-Fixed-Paraffin Embedded (FFPE) samples which require standardization/normalization or qualification of specimen used for analysis. Protein-specific biomarker - accomplished through IHC -may provide less analytical challenges, as compared to e.g. RNA-based biomarker for the purpose of developing technically improved diagnostic test system e.g. such as for the determination of breast cancer molecular subtyping by quantification with the RT-qPCR -based diagnostic system [17]. Sherwood et al. applied the Next Generation Sequencing (NGS) method as a molecular biomarker for the determination of clinically relevant KRAS mutation detection in the Non-Small Cell Lung Cancer (NSCLC) context. The DNA extraction from FFPE tissues though proved to be challenging with respect to ensure an adequate amount of starting DNA while the NGS-based biomarker was developed with extracted DNA from cell lines [18]. Bins et al. in contrast, utilized fresh frozen tumor tissue biopsies to develop the NGS-based biomarker discovery using 469 image guided- biopsies collected in large collaborations in the Netherlands [19]. Nevertheless, the availability of biorepositories, archived human tissues, may provide an added value in early biomarker development, for subsequent (post drug authorization) as well as for concomitantly developing CDx biomarkers for the specific drugs used. Figure 1 illustrates the fundamentally increasing interest in developing skin biomarkers over the last 20 years.

\section{Skin biorepository and biomarker development- regulatory challenges}

Skin cancer has become the most frequent malignant disease in human, while in principle three main types can be categorized: Basal Cell Carcinoma (BCC), Squamous-Cell Carcinoma (SCC) and melanoma, Non-Melanoma Skin Cancer (NMSC) which is the most commonly diagnosed cancer in white-skinned individuals with a worldwide increasing incidence. Merkel Cell Carcinoma (MCC), a consequence of extensive sun exposure coupled with a

\section{Pubmed hits for "Skin Biomarkers" 1996-2017}

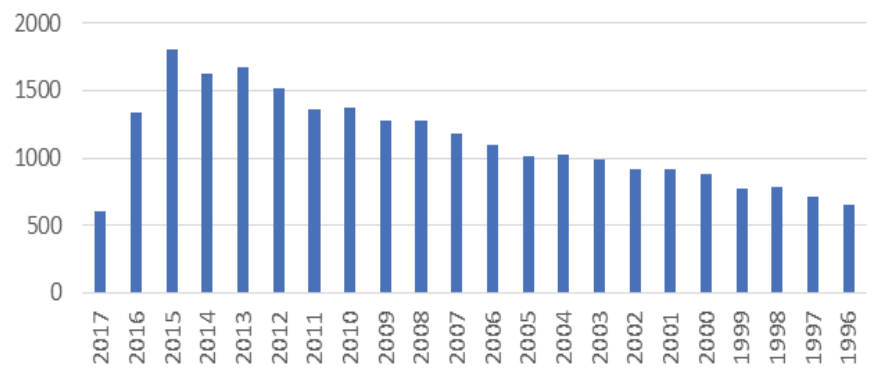

Figure 1 Pubmed hits for "Skin Biomarkers" (year versus number of hits found in Pubmed). 
weak or impaired immune system is a rare but most aggressive skin cancer disease $[20,21]$. Interestingly, the newly discovered polyomavirus, the Merkel Cell polyomavirus was found to be associated in the pathogenesis of MCC. UV induces the DNA damage response involving the tumor suppressor protein p53 [22]. Hess and Brandner [23] found that the polyomavirus, Simian Virus 40 (SV40) modulates wild-type p53 function in SV40transformed cells upon DNA damage and also that p53 plays a central role in DNA-damage induced apoptosis [24]. In addition, virus-transformed cells used as substrates in the manufacture of biologics generally pose a "safety risk" e.g. in the manufacture of vaccines. Thus, polyomaviruses-encoded transforming proteins may play a crucial role in the etiopathogenesis of tumors in various species [25].

The incidence of NMSC has been rising by $3 \%-8 \%$ per year since 1960 , with as much as 300\% increase in the past 2 decades being most likely caused by a combination of ultraviolet (UV) or sunlight, in addition to ozone depletion, genetics and also immuno suppression. Even though there are many approaches to the management of NMSC the current treatment is limited due to low complete clearance rates. Treatment is substantial and increasing, posing a considerable burden on the health care system. As a consequence, there is an urgent need to identify particular biomarkers that can predict this disease and guide the therapeutic treatment options. The use of diagnostic and prognostic biomarkers is of importance in the treatment of melanoma, especially in targeted therapies as illustrated by Weinstein et al. [26].

Biorepository or skin tissue banks allowing early biomarker development. Utilizing legally authorized human-derived tissues certainly may promote and facilitate its development, whenever tissues are available representing targeted skin tissue diseases, such as inflammatory diseases, psoriasis, SCC, BCC or NMSC.

Especially, the development of Immunohistochemistry-based CDx biomarker (IHC) may profit from these biorepositories. Either snap frozen and/or FFPE tissues can be sourced from legally authorized biobanks and tissue suitability testing and subsequent development of performance characteristics and/ or analytical/clinical validation can be performed. Exploratory biomarker development meeting the pre-analytical provisions for CDx development - as stipulated in new European Regulation on in vitro diagnostic medical devices - once Directive 98/79/EC on in-vitro Diagnostic Devices [27] has been repealed in 2022.

This however has become challenging for already existing CDx biomarkers, most of these have been approved by CE-marking

\section{References}

1 On in vitro diagnostic medical devices and repealing Directive 98/79/ EC and Commission Decision 2010/227/EU (2017) Regulation (Eu) $2017 / 746$ of the European Parliament and of the Council.

2 Kamel HFM, Al-Amodi HAS (2017) Exploitation of Gene Expression and Cancer Biomarkers in Paving the Path to Era of Personalized Medicine, Genomics, Proteomics \& Bioinformatics. in a manufacturer self-certification declaration of compliance to Directive 98/79/EC due to the fact that this specific group of CDx, the in-vitro diagnostic medical devices, fall into low risk group as per exclusion classification to Annex II A/B of this Directive. The new regulation classifies $\mathrm{CDx}$ in the second highest risk group (C), which requires the Notified Body (NB) for the conformity assessment approval and for CE-marking. Looking into a 5 year grace period from now on, thereafter all CDx biomarkers approved in the European Union, have to comply with provisions as stipulated in new Regulation on in vitro diagnostic medical devices. This IVDR now strongly emphasizes clinical utility and clinical validity validation not specifically stipulated in "old" (but for the time being still applicable) legislation, the Directive 98/79/EC [28]. Generally, establishing clinical validity for a test system involves showing that the test system is "fit for purpose," a process that relies on data collected from clinical trials or from archived samples that are well annotated with outcomes and anamnestic clinical data. Technical challenges in biomarker test development include lack of standards and reference materials, difficulty in gathering the evidence to assess a test's validity and utility in the clinic, and the need for greater cooperation and sharing, as communicated in a workshop conclusion by Nass et al. [29]. In processing personal data, biobanks assume responsibility for data security. The measures to be taken must safeguard the donors' personality rights for the complete existence of samples and data, that is, from the date when they are collected until the date when they are destroyed [30]. In the world of harmonization and adherence to GCP and to the ethical principles originating in the Declaration of Helsinki [31], there is nevertheless different ethical regulatory frameworks and lack of international consensus which interferes with the efficiency of biomedical research that makes use of biobanks in several countries [32]. These different ethical regulatory frameworks may also be reflected on the level of federal regulations governing human subjects research permitting research on banked tissue without informed consent in several circumstances [33].

As a conclusion, legally, locally authorized biobanks or biorepositories may provide a valuable tool in the early development of performance characteristics and/or analytical/ clinical validation of $C D x$, while the new Regulation on in vitro diagnostic medical devices finally filled in the gaps on regulatory provisions for companion diagnostics biomarker development in the specific context of drug use.

Conflict of interest: Dr. Hess and Dr. Laaff claim a conflict of interest, based on the affiliation of the authors to the company TPL Path Labs.

3 Qualification process for drug development tools (2014) Guidance for Industry and FDA Staff.

4 Biomarkers Definitions Working Group (2001) Biomarkers and surrogate endpoints: preferred definitions and conceptual framework. Clinical Pharmacology and Therapeutics 69: 89-95.

5 Drucker A, Skedgel C, Virik K (2008) The cost burden of trastuzumab and bevacizumab therapy for solid tumours in Canada. Current Oncology 3: 136-142. 
6 Drucker E, Krapfenbauer K (2013) Pitfalls and limitations in translation from biomarker discovery to clinical utility in predictive and personalised medicine. The EPMA J pp: 4-7.

7 Javle M, Hsueh CT (2009) Recent advances in gastrointestinal oncology-updates and insights from the 2009 annual meeting of the American society of clinical oncology. J Hematol Oncol 23: 11-23.

8 Gainor JF, Longo DL, Chabner BA (2014) Pharmacodynamic Biomarkers: Falling Short of the Mark? Clin Cancer Res 20: 25872594.

9 OECD (2011) Policy Issues for the Development and Use of Biomarkers in Health.

10 Lv Q, Meng Z, Yu Y (2016) Molecular Mechanisms and Translational Therapies for Human Epidermal Receptor 2 Positive Breast Cancer. Int J Mol Sci 17: 2095.

11 Ross JS, Fletcher JA, Linette GP (2003) The HER-2/neu Gene and Protein in Breast Cancer 2003: Biomarker and Target of Therapy. Oncologist 8: 307-325.

12 http://www.ema.europa.eu/ema/index.jsp?curl=pages/ medicines/human/medicines/000278/human_med_000818. jsp\&mid=WC0b01ac058001d124

13 OECD (2007) OECD Best Practice guidelines for Biological resource centers.

14 http://www.ema.europa.eu/docs/en_GB/document_library/ Regulatory_and_procedural_guideline/2012/05/WC500127124.pdf

15 Guideline on the evaluation of anticancer medicinal products in man (2012) European medicines agency, Science medicines health.

16 Simon RM, Paik S, Hayes DF (2009) Use of Archived Specimens in Evaluation of Prognostic and Predictive Biomarkers. JNCl.

17 Laible M, Schlombs K, Kaiser K (2016) Technical validation of an RTqPCR in vitro diagnostic test system for the determination of breast cancer molecular subtypes by quantification of ERBB2, ESR1, PGR and MKI67 mRNA levels from formalin fixed paraffin-embedded breast tumor specimens. BMC Cancer 16: 398.

18 Sherwood JL, Brown H, Rettino A (2017) Key differences between 13 KRAS mutation detection technologies and their relevance for clinical practice.

19 Bins S, Cirkel GA, Gadellaa-Van Hooijdonk CG (2017) Implementation of a Multicenter Biobanking Collaboration for Next-Generation
Sequencing-Based Biomarker Discovery Based on Fresh Frozen Pretreatment Tumor Tissue Biopsies. Oncologist 22: 33-40.

20 Wang L, Xu J, Duan B (2017) Association between polymorphisms in DNA repair gene XRCC1 and non-melanoma skin cancer risk: a metaanalysis. Onco Targets Ther 10: 3475-3483.

21 Haymerle G, Janik S, Fochtmann A (2017) Expression of Merkelcell polyomavirus (MCPyV) large T-antigen in Merkel cell carcinoma lymph node metastases predicts poor outcome. Plos one 12 : e0180426.

22 Hess R, Plaumann B, Schulze Lutum A (1994) Nuclear accumulation of p53 in response to treatment with DNA-damaging agents. Toxicol Letters 72: 43-52.

23 Hess RD, Brandner G (1997) DNA-damage-inducible p53 activity in SV40-transformed cells. Oncogene 15: 2401-2501.

24 Hess RD, Brandner G (1996) P53 in normal and neoplastic tissue: analysis and function. El J Pathol Histol 961-1007.

25 Hess RD, Weber F, Watson K (2012) Regulatory, biosafety and safety challenges for novel cells as substrates for human vaccines. Vaccine 30: 2715-2727.

26 Weinstein D, Leininger J, Hamby C (2014) Diagnostic and Prognostic Biomarkers in Melanoma. J Clin Aesthet Dermatol 7: 13-24.

27 On in vitro diagnostic medical devices (1998) Directive 98/79/EC of the european parliament and of the council.

28 Hess RD (2012) Biomarker: Regulierung der In-vitro Diagnostika in Europa: Herausforderungen der Zukunft, begleitdiagnostische Verfahren. Forum Institut Fur Management GmbH Newsletter.

29 Nass SJ, Phillips J, Patlak M (2015) Policy Issues in the Development and Adoption of Biomarkers for Molecularly Targeted Cancer Therapies: Workshop Summary. National Cancer Policy Forum; Board on Health Care Services; Institute of Medicine.

30 http://www.ethikrat.org/files/der_opinion_human-biobanks.pdf

31 http://www.ich.org/fileadmin/Public_Web_Site/ICH_Products/ Guidelines/Efficacy/E6/E6_R2_Addendum_Step2.pdf

32 Elger BS, Kaplan AL (2006) Consent and anonymization in research involving biobanks. Differing terms and norms present serious barriers to an international framework EMBO Rep. 7: 661-666.

33 Botkin JR (2012) Informed Consent for Biobank-Dependent Research. AMA J Ethics 14: 610-615. 